\title{
CERTAIN INTEGRALS ASSOCIATED WITH GENERALIZED HYPERGEOMETRIC FUNCTIONS
}

\author{
D.L. Suthar, S.D. Purohit, K.S. Nisar
}

ABstract. The present paper deals with the three new integral formulas involving the extended generalized hypergeometric function and are expressed in term of the generalized hypergeometric functions. Some important particular cases involving the extended Gauss hypergeometric and confluent hypergeometric functions are also pointed out.

2010 Mathematics Subject Classification: 33B15, 33B20, 33C05, 33C20.

Keywords: Generalized hypergeometric function, Gamma function, Lavoie-Trottier integral formula, Generalized Pochhammer symbol.

\section{INTRODUCTION}

Recently, Srivastava et al. [11] studied and established in a methodical manner the following extended generalized hypergeometric function:

$$
{ }_{r} F_{s}\left[\begin{array}{c}
\left(\delta_{1}, m\right), \delta_{2}, \ldots, \delta_{r} ; \\
\xi_{1}, \ldots, \xi_{s} ;
\end{array}\right]=\sum_{p=0}^{\infty} \frac{\left(\delta_{1}, m\right)_{p}\left(\delta_{2}\right)_{p}, \ldots,\left(\delta_{r}\right)_{p}}{\left(\xi_{1}\right)_{p}, \ldots,\left(\xi_{s}\right)_{p}} \frac{(x)^{p}}{p !}
$$

where $(\lambda ; m)_{\nu}$ denotes the Pochhammer symbol defined, as follow (see [11], p. 485 Eq.(1.66))

$$
(\lambda ; m)_{\nu}=\left\{\begin{array}{cl}
\frac{\Gamma_{m}(\lambda+\nu)}{\Gamma(\lambda)}, & (\Re(m)>0 ; \nu, \lambda \in \mathbb{C}), \\
(\lambda)_{\nu}, & (m=0 ; \nu, \lambda \in \mathbb{C}) .
\end{array}\right.
$$

Here, Chaudhary and Zubair ([1], p. 9, eq.(1.66)) is introduced the generalized gamma function as follows:

$$
\Gamma_{m}(z)=\left\{\begin{array}{cc}
\int_{0}^{\infty} t^{z-1} \exp (-t-m / t) d t, & (\Re(m)>0 ; z \in \mathbb{C}), \\
\Gamma(z), & (m=0 ; \Re(z)>0) .
\end{array}\right.
$$


The corresponding extensions of Gauss's hypergeometric and confluent hypergeometric function are as follows:

$$
{ }_{2} F_{1}\left[\begin{array}{cc}
(\delta, m), \xi ; & x \\
\gamma ; &
\end{array}\right]=\sum_{p=0}^{\infty} \frac{(\delta, m)_{p}(\xi)_{p}}{(\gamma)_{p}} \frac{(x)^{p}}{p !},
$$

and

$$
{ }_{1} F_{1}\left[\begin{array}{c}
(\delta, m) ; \\
\gamma ;
\end{array}\right]=\sum_{n=0}^{\infty} \frac{(\delta, m)_{p}}{(\gamma)_{p}} \frac{(x)^{p}}{p !} .
$$

Recently, the integral representations of various special functions are studied widely (see $[4,5,6,7,8,9,12,13,14])$. Here, we aim to find the integral and double integral representations of generalized hypergeometric function. To approach toward our main findings, we recall the following Lavoie-Trottier integral formula [3]

$$
\int_{0}^{1} x^{\vartheta-1}(1-x)^{2 \tau-1}\left(1-\frac{x}{3}\right)^{2 \vartheta-1}\left(1-\frac{x}{4}\right)^{\tau-1} d x=\left(\frac{2}{3}\right)^{2 \vartheta} \frac{\Gamma(\vartheta) \Gamma(\tau)}{\Gamma(\vartheta+\tau)}
$$

provided $\Re(\vartheta)>0, \Re(\tau)>0$.

Also, we need the Edward integral formula [2, 7, p.445; see also p.243]:

$$
\int_{0}^{1} \int_{0}^{1} y^{\vartheta}(1-x)^{\vartheta-1}(1-y)^{\tau-1}(1-x y)^{1-\vartheta-\tau} d x d y=\frac{\Gamma(\vartheta) \Gamma(\tau)}{\Gamma(\vartheta+\tau)}, 0<\Re(\tau)<\Re(\vartheta) .
$$

\section{Main Results}

We establish three generalized integral formulas, which are represented in terms of the generalized hypergeometric functions (1) by inserting with the suitable argument in the integrand of (6) and (7).

Theorem 1. Let $x>0, \alpha_{1}, \alpha_{2}, \ldots, \alpha_{r}, \delta, \eta \in \mathbb{C}$; and $\beta_{1}, \ldots, \beta_{s} \in \mathbb{C} \backslash \mathbb{Z}_{0}$ with $\Re(m)>0, \Re(\delta)>0, \Re(\eta)>0, \Re(\delta+\eta)>0$, then the following result holds:

$$
\begin{aligned}
& \int_{0}^{\infty} x^{\delta-1}(1-x)^{2 \eta-1}\left(1-\frac{x}{3}\right)^{2 \delta-1}\left(1-\frac{x}{4}\right)^{\eta-1}
\end{aligned}
$$

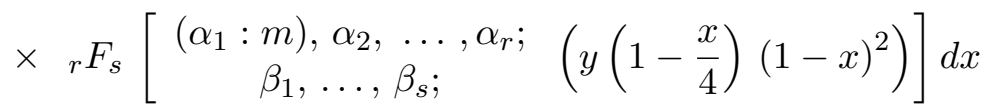

$$
\begin{aligned}
& =\left(\frac{2}{3}\right)^{2 \delta} \frac{\Gamma(\delta) \Gamma(\eta)}{\Gamma(\delta+\eta)} r_{+1} F_{s+1}\left[\begin{array}{c}
\left(\alpha_{1}: m\right), \alpha_{2}, \ldots, \alpha_{r}, \eta ; \\
\beta_{1}, \ldots, \beta_{s}, \delta+\eta ;
\end{array}\right] .
\end{aligned}
$$


Proof. Let $\mathfrak{S}_{1}$ be the left-hand side (LHS) of (8). Using (1) in the integrand of (8) and then interchanging the order of integration and summation, which is valid under the given conditions, we get

$$
\begin{gathered}
\mathfrak{S}_{1}=\sum_{p=0}^{\infty} \frac{\left(\alpha_{1}: m\right)_{p}\left(\alpha_{2}\right)_{p} \ldots\left(\alpha_{r}\right)_{p}}{\left(\beta_{1}\right)_{p} \ldots\left(\beta_{s}\right)_{p} n !} y^{p} \\
\times \int_{0}^{1} x^{\delta-1}(1-x)^{2(\eta+p)-1}\left(1-\frac{x}{3}\right)^{2 \delta-1}\left(1-\frac{x}{4}\right)^{\eta+p-1} d x
\end{gathered}
$$

Now, on using the integral formula (6), the above integral (9) can be written as:

$$
\begin{gathered}
\mathfrak{S}_{\mathbf{1}}=\sum_{p=0}^{\infty} \frac{\left(\alpha_{1}: m\right)_{p}\left(\alpha_{2}\right)_{p} \ldots\left(\alpha_{r}\right)_{p}}{\left(\beta_{1}\right)_{p} \ldots\left(\beta_{s}\right)_{p} p !} y^{p}\left(\frac{2}{3}\right)^{2 \delta} \frac{\Gamma(\delta) \Gamma(\eta+p)}{\Gamma(\delta+\eta+p)}, \\
=\left(\frac{2}{3}\right)^{2 \delta} \frac{\Gamma(\delta) \Gamma(\eta)}{\Gamma(\delta+\eta)} \sum_{p=0}^{\infty} \frac{\left(\alpha_{1}: m\right)_{p}\left(\alpha_{2}\right)_{p} \ldots\left(\alpha_{r}\right)_{p}}{\left(\beta_{1}\right)_{p} \ldots\left(\beta_{s}\right)_{p}} \frac{(\eta)_{p}}{(\delta+\eta)_{p} p !}(y)^{p},
\end{gathered}
$$

In accordance with the definition of (1), we easily obtain the result (8).

Theorem 2. Let $x>0, \alpha_{1}, \alpha_{2}, \ldots, \alpha_{r}, \delta, \eta \in \mathbb{C}$; and $\beta_{1}, \ldots, \beta_{s} \in \mathbb{C} \backslash \mathbb{Z}_{0}$ with $\Re(m)>0, \Re(\delta)>0, \Re(\eta)>0, \Re(\delta+\eta)>0$, then

$$
\begin{aligned}
& \int_{0}^{\infty} x^{\delta-1}(1-x)^{2 \eta-1}\left(1-\frac{x}{3}\right)^{2 \delta-1}\left(1-\frac{x}{4}\right)^{\eta-1} \\
& \times{ }_{r} F_{s}\left[\begin{array}{c}
\left(\alpha_{1}: p\right), \alpha_{2}, \ldots, \alpha_{r} ; \\
\beta_{1}, \ldots, \beta_{s} ;
\end{array} y x\left(1-\frac{x}{3}\right)^{2}\right] d x \\
& =\left(\frac{2}{3}\right)^{2 \delta} \frac{\Gamma(\delta) \Gamma(\eta)}{\Gamma(\delta+\eta)} r+1 F_{s+1}\left[\begin{array}{cc}
\left(\alpha_{1}: p\right), \alpha_{2}, \ldots, \alpha_{r}, \delta ; & \frac{4 y}{9} \\
\beta_{1}, \ldots, \beta_{s}, \delta+\eta ; & .
\end{array}\right] .
\end{aligned}
$$

Proof. Denoting the LHS of (10) by $\mathfrak{S}_{2}$. Now, we make use of (1) in the integrand of (10), and then interchange the order of integration and summation, which is valid under the given conditions, to obtain

$$
\begin{gathered}
\mathfrak{S}_{2}=\sum_{p=0}^{\infty} \frac{\left(\alpha_{1}: m\right)_{p}\left(\alpha_{2}\right)_{p} \ldots\left(\alpha_{r}\right)_{p}}{\left(\beta_{1}\right)_{p} \ldots\left(\beta_{s}\right)_{p} p !} y^{p} \\
\times \int_{0}^{1} x^{\delta+p-1}(1-x)^{2 \eta-1}\left(1-\frac{x}{3}\right)^{2(\delta+p)-1}\left(1-\frac{x}{4}\right)^{\eta-1} d x
\end{gathered}
$$


which on using integral formula (6) arrive at

$$
\begin{gathered}
\mathfrak{S}_{2}=\sum_{p=0}^{\infty} \frac{\left(\alpha_{1}: m\right)_{p}\left(\alpha_{2}\right)_{p} \ldots\left(\alpha_{r}\right)_{p}}{\left(\beta_{1}\right)_{p} \ldots\left(\beta_{s}\right)_{p} p !} y^{p}\left(\frac{2}{3}\right)^{2(\delta+p)} \frac{\Gamma(\delta+p) \Gamma(\eta)}{\Gamma(\delta+\eta+p)}, \\
=\left(\frac{2}{3}\right)^{2 \delta} \frac{\Gamma(\delta) \Gamma(\eta)}{\Gamma(\delta+\eta)} \sum_{p=0}^{\infty} \frac{\left(\alpha_{1}: m\right)_{p}\left(\alpha_{2}\right)_{p} \ldots\left(\alpha_{r}\right)_{p}}{\left(\beta_{1}\right)_{p} \ldots\left(\beta_{s}\right)_{p}} \frac{(\delta)_{p}}{(\delta+\eta)_{p} p !}\left(\frac{4 y}{9}\right)^{p} .
\end{gathered}
$$

Now, in accordance with the definition of (1), we obtain the result (10).

Remark 1. In Theorems 1 and 2, by making some suitable adjustment of the parameters, we arrive at the known result given by Parmar and Purohit ([10]).

Now, we derive double integral involving generalized hypergeometric function using the formula (7).

Theorem 3. Let $x>0, \alpha_{1}, \alpha_{2}, \cdots, \alpha_{r}, \lambda, \mu \in \mathbb{C}$ and $\beta_{1}, \cdots, \beta_{s} \in \mathbb{C} \backslash \mathbb{Z}_{0}$ with $\Re(\mu)>0,0<\Re(\mu)<\Re(\lambda)$, then the following formula hold true.

$$
\begin{gathered}
\int_{0}^{1} \int_{0}^{1} y^{\lambda}(1-x)^{\lambda-1}(1-y)^{\mu-1}(1-x y)^{1-\lambda-\mu} \\
\times_{r} F_{s}\left[\begin{array}{cc}
\left(\alpha_{1}, m\right), \alpha_{2}, \ldots, \alpha_{r} ; & \frac{a y(1-x)(1-y)}{(1-x y)^{2}} \\
\beta_{1}, \ldots, \beta_{s} ;
\end{array}\right] \\
=\frac{\Gamma(\lambda) \Gamma(\mu)}{\Gamma(\lambda+\mu)} \quad{ }_{r+2} F_{s+2}\left[\begin{array}{cc}
\left(\alpha_{1}, m\right), \alpha_{2}, \ldots, \alpha_{r}, \lambda, \mu ; & a \\
\beta_{1}, \ldots, \beta_{s}, \frac{\lambda+\mu}{2}, \frac{\lambda+\mu+1}{2} ; & \frac{a}{4}
\end{array}\right] .
\end{gathered}
$$

Proof. Let the left hand side be denoted by $\mathfrak{I}$, then

$$
\begin{aligned}
\mathfrak{I}= & \int_{0}^{1} \int_{0}^{1} y^{\lambda}(1-x)^{\lambda-1}(1-y)^{\mu-1}(1-x y)^{1-\lambda-\mu} \\
& \times{ }_{r} F_{s}\left[\begin{array}{c}
\left(\alpha_{1}, m\right), \alpha_{2}, \ldots, \alpha_{r} ; \\
\beta_{1}, \ldots, \beta_{s} ;
\end{array} \frac{a y(1-x)(1-y)}{(1-x y)^{2}}\right],
\end{aligned}
$$

Now, on using the definition (1), we get

$$
\begin{aligned}
\mathfrak{I} & =\int_{0}^{1} \int_{0}^{1} y^{\lambda}(1-x)^{\lambda-1}(1-y)^{\mu-1}(1-x y)^{1-\lambda-\mu} \\
& \times \sum_{p=0}^{\infty} \frac{\left(\alpha_{1}, m\right)_{p},\left(\alpha_{2}\right)_{p}, \ldots,\left(\alpha_{r}\right)_{p}}{(\beta 1)_{p}, \ldots,\left(\beta_{s}\right)_{p} p !} \frac{a^{p} y^{p}(1-x)^{p}(1-y)^{p}}{(1-x y)^{2 p}} d x d y,
\end{aligned}
$$


which on changing the order of integration and summation arrive at

$$
\begin{aligned}
\mathfrak{I} & =\sum_{p=0}^{\infty} \frac{\left(\alpha_{1}, m\right)_{p},\left(\alpha_{2}\right)_{p}, \ldots,\left(\alpha_{r}\right)_{p}}{(\beta 1)_{p}, \ldots,\left(\beta_{s}\right)_{p} p !} a^{p} \\
& \times \int_{0}^{1} \int_{0}^{1} y^{p+\lambda}(1-x)^{p+\lambda-1}(1-y)^{p+\mu-1}(1-x y)^{1-\lambda-\mu-2 p} d x d y,
\end{aligned}
$$

Further, on applying the integral formula given (7), we get

$$
\mathfrak{I}=\sum_{p=0}^{\infty} \frac{\left(\alpha_{1}, m\right)_{p},\left(\alpha_{2}\right)_{p}, \ldots,\left(\alpha_{r}\right)_{p}}{(\beta 1)_{p}, \ldots,\left(\beta_{s}\right)_{p} p !} a^{p} \frac{\Gamma(\lambda+p) \Gamma(\mu+p)}{\Gamma(\lambda+\mu+2 p)},
$$

and then by using the formula,

$$
(\lambda)_{k n}=k^{k n}\left(\frac{\lambda}{k}\right)_{n}\left(\frac{\lambda+1}{k}\right)_{n} \ldots\left(\frac{\lambda+k-1}{k}\right)_{n},
$$

we obtain

$$
\begin{aligned}
\mathfrak{I} & =\frac{\Gamma(\lambda+p) \Gamma(\mu+p)}{\Gamma(\lambda+\mu+2 p)} \sum_{p=0}^{\infty} \frac{\left(\alpha_{1}, m\right)_{p},\left(\alpha_{2}\right)_{p}, \ldots,\left(\alpha_{r}\right)-p(\lambda)_{p}(\mu)_{p}}{(\beta 1)_{p}, \ldots,\left(\beta_{s}\right)_{p} p !} \\
& \times\left(\frac{\lambda+\mu}{2}\right)_{p}\left(\frac{\lambda+\mu+1}{2}\right)_{p}\left(\frac{a}{4}\right)^{p},
\end{aligned}
$$

In view of (1), we arrived at the desired result.

\section{Special Cases}

Some important particular cases of the main theorems are considered in this section. We present certain integrals as Corollaries, given below for extended Gauss hypergeometric and confluent hypergeometric functions (4) and (5).

Corollary 4. Let $x>0, \alpha, \beta, \delta, \eta \in \mathbb{C}$; and $\gamma \in \mathbb{C} \backslash \mathbb{Z}_{0}$ with $\Re(m)>0, \Re(\delta)>$ $0, \Re(\eta)>0, \Re(\delta+\eta)>0$, then there hold the following results:

$$
\begin{aligned}
& \int_{0}^{\infty} x^{\delta-1}(1-x)^{2 \eta-1}\left(1-\frac{x}{3}\right)^{2 \delta-1}\left(1-\frac{x}{4}\right)^{\eta-1} \\
& \times{ }_{r} F_{s}\left[\begin{array}{c}
(\alpha: m), \beta ; \\
\gamma ;
\end{array}\left(y\left(1-\frac{x}{4}\right)(1-x)^{2}\right)\right] d x
\end{aligned}
$$




$$
=\left(\frac{2}{3}\right)^{2 \delta} \frac{\Gamma(\delta) \Gamma(\eta)}{\Gamma(\delta+\eta)}{ }_{3} F_{2}\left[\begin{array}{c}
(\alpha: m), \beta, \eta ; \\
\gamma, \delta+\eta ;
\end{array}\right] .
$$

Corollary 5. Let $x>0, \alpha, \beta, \delta, \eta \in \mathbb{C}$; and $\gamma \in \mathbb{C} \backslash \mathbb{Z}_{0}$ with $\Re(m)>0, \Re(\delta)>0$, $\Re(\eta)>0, \Re(\delta+\eta)>0$, then there hold the following results:

$$
\begin{aligned}
& \int_{0}^{\infty} x^{\delta-1}(1-x)^{2 \eta-1}\left(1-\frac{x}{3}\right)^{2 \delta-1}\left(1-\frac{x}{4}\right)^{\eta-1}
\end{aligned}
$$

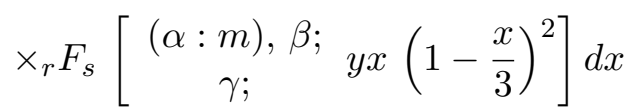

$$
\begin{aligned}
& =\left(\frac{2}{3}\right)^{2 \delta} \frac{\Gamma(\delta) \Gamma(\eta)}{\Gamma(\delta+\eta)}{ }_{3} F_{2}\left[\begin{array}{cc}
(\alpha: m), \beta, \delta ; & \frac{4 y}{9} \\
\gamma, \delta+\eta ; & .
\end{array}\right.
\end{aligned}
$$

Corollary 6. Let $x>0, \alpha, \beta, \lambda, \mu \in \mathbb{C}$ and $\beta_{1}, \cdots, \beta_{r} \mathbb{C} \backslash \mathbb{Z}_{0}$ with $\Re(\mu)>0,0<$ $\Re(\mu)<\Re(\lambda)$, then the following formula hold true.

$$
\begin{gathered}
\int_{0}^{1} \int_{0}^{1} y^{\lambda}(1-x)^{\lambda-1} \\
(1-y)^{\mu-1}(1-x y)^{1-\lambda-\mu}{ }_{2} F_{1}\left[\begin{array}{cc}
(\alpha, m), \beta ; & \frac{a y(1-x)(1-y)}{(1-x y)^{2}}
\end{array}\right] d x d y \\
=\frac{\Gamma(\lambda) \Gamma(\mu)}{\Gamma(\lambda+\mu)} \quad{ }_{4} F_{3}\left[\begin{array}{cc}
(\alpha, m), \beta, \lambda, \mu ; & a \\
\gamma, \frac{\lambda+\mu}{2}, \frac{\lambda+\mu+1}{2} ; & \frac{a}{4}
\end{array}\right] .
\end{gathered}
$$

Corollary 7. Let $x>0, \alpha, \delta, \eta \in \mathbb{C}$; and $\gamma \in \mathbb{C} \backslash \mathbb{Z}_{0}$ with $\Re(m)>0, \Re(\delta)>0$, $\operatorname{Re}(\eta)>0, \Re(\delta+\eta)>0$, then there hold the following results:

$$
\begin{aligned}
& \int_{0}^{\infty} x^{\delta-1}(1-x)^{2 \eta-1}\left(1-\frac{x}{3}\right)^{2 \delta-1}\left(1-\frac{x}{4}\right)^{\eta-1} \\
& \times{ }_{r} F_{s}\left[\begin{array}{c}
\left.(\alpha: m) ; \quad\left(y\left(1-\frac{x}{4}\right)(1-x)^{2}\right)\right] d x \\
\gamma ;
\end{array}\right. \\
& =\left(\frac{2}{3}\right)^{2 \delta} \frac{\Gamma(\delta) \Gamma(\eta)}{\Gamma(\delta+\eta)}{ }_{2} F_{2}\left[\begin{array}{c}
(\alpha: m), \eta ; \\
\gamma, \delta+\eta ;
\end{array}\right] .
\end{aligned}
$$

Corollary 8. Let $x>0, \alpha, \delta, \eta \in \mathbb{C}$; and $\gamma \in \mathbb{C} \backslash \mathbb{Z}_{0}$ with $\Re(m)>0, \Re(\delta)>0$, $\Re(\eta)>0, \Re(\delta+\eta)>0$, then there hold the following results:

$$
\int_{0}^{\infty} x^{\delta-1}(1-x)^{2 \eta-1}\left(1-\frac{x}{3}\right)^{2 \delta-1}\left(1-\frac{x}{4}\right)^{\eta-1}{ }_{r} F_{s}\left[\begin{array}{c}
(\alpha: m) ; \\
\gamma ;
\end{array} y\left(1-\frac{x}{3}\right)^{2}\right] d x
$$




$$
=\left(\frac{2}{3}\right)^{2 \delta} \frac{\Gamma(\delta) \Gamma(\eta)}{\Gamma(\delta+\eta)}{ }_{2} F_{2}\left[\begin{array}{cc}
(\alpha: m), \delta ; & \frac{4 y}{9} \\
\gamma, \delta+\eta ; & .
\end{array}\right.
$$

Corollary 9. Let $x>0, \alpha, \beta, \lambda, \mu \in \mathbb{C}$ and $\beta_{1}, \cdots, \beta_{r} \mathbb{C} \backslash \mathbb{Z}_{0}$ with $\Re(\mu)>0,0<$ $\Re(\mu)<\Re(\lambda)$, then the following formula hold true.

$$
\begin{gathered}
\int_{0}^{1} \int_{0}^{1} y^{\lambda}(1-x)^{\lambda-1}(1-y)^{\mu-1}(1-x y)^{1-\lambda-\mu}{ }_{2} F_{1}\left[\begin{array}{cc}
(\alpha, m) ; & \frac{a y(1-x)(1-y)}{(1-x y)^{2}}
\end{array}\right] d x d y \\
\gamma ; \\
=\frac{\Gamma(\lambda) \Gamma(\mu)}{\Gamma(\lambda+\mu)} \quad{ }_{4} F_{3}\left[\begin{array}{cc}
(\alpha, m), \lambda, \mu ; & a \\
\gamma, \frac{\lambda+\mu}{2}, \frac{\lambda+\mu+1}{2} ; & \frac{a}{4}
\end{array}\right] .
\end{gathered}
$$

\section{REFERENCES}

[1] M.A. Chaudhry and S.M. Zubair, On a class of incomplete gamma functions with applications, Chapman \& Hall/CRC, Boca Raton, FL, (2002).

[2] J. Edward, A Treatise on the Integral Calculus, Vol. II, Chelsea Publishing Company, New York, (1922).

[3] J.L. Lavoie and G. Trottier, On the sum of certain Appell series, Ganita, 20(1)(1969), 43-66.

[4] N. Menaria, K.S. Nisar and S.D. Purohit, On a new class of integrals involving product of generalized Bessel function of the first kind and general class of polynomials, Acta Univ. Apulensis Math. Inform., 46(2016), 97-105.

[5] N. Menaria, R.K. Parmar, S.D. Purohit and K.S. Nisar, Certain unified integrals involving product of generalized k-Bessel function and general class of polynomials, Honam Mathematical J., 39(3)(2017), 349-361.

[6] S.R. Mondal and K.S. Nisar, Certain unified integral formulas involving the generalized modified $k$-Bessel function of first kind, Commun. Korean Math. Soc., $32(1)(2017), 47-53$.

[7] K.S. Nisar and S.R. Mondal, Some integrals involving generalized $k$-Struve functions, Commun. Numer. Anal., 2017(1)(2017), 19-24.

[8] K.S. Nisar, R.K. Parmar and A.H. Abusufian, Certain new unified integrals associated with the generalized $k$-Bessel function, Far East J. Appl. Math., 100(9)(2016), 1357-1544.

[9] K.S. Nisar, D.L. Suthar, S.D. Purohit and M. Aldhaifallah, Some unified integrals associated with generalized Struve function, Proc. Jangjeon Math. Soc., 20(2)(2017), 261-267. 
D.L. Suthar, S.D. Purohit, K.S. Nisar - Certain integrals...

[10] R.K. Parmar and S.D. Purohit, On a new class of integrals involving generalized hypergeometric function, Int. Bull. Math. Res., 3(2016), 24-27.

[11] H.M. Srivastava, A. Cetinkaya and I. Onur Kiyamaz, A certain generalized pochhammer symbol and its applications to hypergeometric functions, Appl. Math. Comput., 226(2014), 484-491.

[12] D.L. Suthar, H. Habenom and H. Tadesse, Certain integrals involving Aleph function and Wright's generalized hypergeometric function, Acta Univ. Apulensis Math. Inform., 52(4)(2017), 1-10.

[13] D.L. Suthar, S.D. Purohit and S. Agarwal, Class of integrals involving generalized hypergeometric function and Srivastava's polynomials, Int. J. Appl. Comput. Math., 3(Suppl-1)(2017), S1197-S1203.

[14] D.L. Suthar, S.D. Purohit and K.S. Nisar, Integral transforms of the Galue type Struve function, TWMS J. App. Eng. Math., 8(1)(2018), 114-121.

\section{D.L. Suthar}

Department of Mathematics,

Wollo University,

Dessie, Ethiopia

email:dlsuthar@gmail.com

S.D. Purohit

Department of HEAS (Mathematics),

Rajasthan Technical University,

Kota, India

email:sunil_a_purohit@yahoo.com

K.S. Nisar

Department of Mathematics, College of Arts \& Science-Wadi Al-Dawaser, Prince Sattam bin Abdulaziz University,

Alkhatj, Saudi Arabia

email: ksnisar1@gmail.com 